\title{
ABOUT CONVERGENCE RATES IN REGULARIZATION FOR ILL-POSED OPERATOR EQUATIONS OF HAMMERSTEIN TYPE
}

\author{
NGUYEN BUONG ${ }^{1}$, DANG THI HAI HA ${ }^{2}$ \\ ${ }^{1}$ Vietnamse Academy of Science and Technology, Institute of Information Technology \\ ${ }^{2}$ Vietnamese Forestry University, Xuan Mai, Ha Tay
}

\begin{abstract}
The aim of this paper is to study convergence rates of the regularized solutions in connection with the finite-dimensional approximations for the operator equation of Hammerstein type $x+F_{2} F_{1}(x)=f$ in reflexive Banach spaces under the perturbations for not only the operators $F_{i}, i=1,2$, but also $f$. The conditions of convergence and convergence rates given in this paper for a class of inverse-strongly monotone operators $F_{i}, i=1,2$, are much simpler than those in the past papers.

Tóm tắt. Mục đích của bài báo này là nghiên cứu tốc độ hội tụ của nghiệm hiệu chinh đã được xấp xỉ hữu hạn chiều cho phương trình toán tử loại Hammerstein $x+F_{2} F_{1}(x)=f$ trong không gian Banach phản xạ với nhiễu không chỉ có ở các toán tử $F_{i}, i=1,2$ mà cả ở $f$. Điều kiện hội tụ và tốc độ hội tụ trong bài báo này cho toán tử ngược đơn điệu mạnh $F_{i}, i=1,2$ là yếu hơn nhiều so với các kết quả trước.
\end{abstract}

\section{INTRODUCTION}

Let $X$ be a reflexive real Banach space, and $X^{*}$ be its dual which both are strictly convex. For the sake of simplicity the norms of $X$ and $X^{*}$ are denoted by the symbol $\|$.$\| . We write$ $\left\langle x^{*}, x\right\rangle$ or $\left\langle x, x^{*}\right\rangle$ instead of $x^{*}(x)$ for $x^{*} \in X^{*}$ and $x \in X$. Concerning the space $X$, in addition assume that it possesses the property: the weak convergence and convergence of norms for any sequence follows its strong convergence. Let $F_{1}: X \rightarrow X^{*}$ and $F_{2}: X^{*} \rightarrow X$ be monotone, in general nonlinear, bounded (i.e. image of any bounded subset is bounded) and continuous operators.

Our main aim of this paper is to study a stable method of finding an approximative solution for the equation of Hammerstein type

$$
x+F_{2} F_{1}(x)=f, f \in X .
$$

Usually instead of $F_{i}, i=1,2$, and $f$ we know their monotone continuous approximations $F_{i}^{h}$ and $f_{\delta}$, such that

$$
\begin{gathered}
\left\|F_{1}^{h}(x)-F_{1}(x)\right\| \leqslant h g(\|x\|) \quad \forall x \in X, \\
\left\|F_{2}^{h}\left(x^{*}\right)-F_{2}\left(x^{*}\right)\right\| \leqslant h g\left(\left\|x^{*}\right\|\right) \quad \forall x^{*} \in X^{*}, \\
g(t) \leqslant M t+N, \quad M, N \geqslant 0
\end{gathered}
$$


where $g(t)$ is a real nonegative, non-decreasing, bounded function (the image of a bounded set is bounded) with $g(0)=0$, and $\left\|f_{\delta}-f\right\| \leqslant \delta$. Without additional conditions for the operators $F_{i}$ such as the strongly monotone property, equation (1.1) is ill-posed (see the example at the end of the paper). To solve (1.1) we need to use stable methods. One of them is the operator equation

$$
x+F_{2, \alpha}^{h} F_{1, \alpha}^{h}(x)=f_{\delta}
$$

(see [1], [2]), where $F_{i, \alpha}^{h}=F_{i}^{h}+\alpha U_{i}, U_{i}, i=1,2$, are the normalized dual mappings of $X$ and $X^{*}$, respectively (see [9]), and $\alpha>0$ is the small parameter of regularization. For every $\alpha>0$ equation (1.2) has a unique solution $x_{\alpha}^{h, \delta}$, and the sequence $\left\{x_{\alpha}^{h, \delta}\right\}$ converges to a solution $x_{0}$ of $(1.1)$ as $(h+\delta) / \alpha, \alpha \rightarrow 0$. Moreover, this solution $x_{\alpha}^{h, \delta}$, for every fixed $\alpha>0$, depends continuously on $F_{i}^{h}, i=1,2$ and $f_{\delta}$, the finite-dimensional problems

$$
x+F_{2, \alpha, n}^{h} F_{1, \alpha, n}^{h}(x)=f_{\delta, n}, x \in X_{n},
$$

where $F_{2, \alpha, n}^{h}=P_{n} F_{2, \alpha}^{h} P_{n}^{*}, F_{1, \alpha, n}^{h}=P_{n}^{*} F_{1, \alpha}^{h} P_{n}, f_{\delta, n}=P_{n} f_{\delta}, P_{n}$ is a linear projection from $X$ onto its finite-dimensional subspace $X_{n}$ such that $X_{n} \subset X_{n+1}, P_{n} x \rightarrow x$, as $n \rightarrow \infty$ for every $x \in X$, and $P_{n}^{*}$ is the dual of $P_{n}$ with $\left\|P_{n}\right\| \leqslant \tilde{c}=$ constant, for all $n$, have a unique solution $x_{\alpha, n}^{h, \delta}$, and the sequence $\left\{x_{\alpha, n}^{h, \delta}\right\}$ converges to $x_{\alpha}^{h, \delta}$, as $n \rightarrow \infty$, without additional conditions on $F_{i}, i=1,2$. In the case of linearity for $F_{2}$ and $f_{\delta}=f$ for all $\delta>0$, the convergence rates for the sequences $\left\{x_{\alpha}^{h, \delta}\right\}$ and $\left\{x_{\alpha, n}^{h, \delta}\right\}$ are given in the paper [3] provided the existence of bounded inversion $\left(I+F_{2} F_{1}^{\prime}\left(x_{0}\right)\right)^{-1}$, where $I$ denotes the identity operator in $X$. It is not difficult to verify that this condition can be replaced by the bounded inversion of $\left(I+F_{2}^{\prime}\left(x_{0}^{*}\right) F_{1}^{\prime}\left(x_{0}\right)\right)^{-1}$, when $F_{2}$ also is nonlinear, where $x_{0}^{*}=F_{1}\left(x_{0}\right)$. The last requirement is equivalent to that -1 is not an eigenvalue of the operator $F_{2}^{\prime}\left(x_{0}^{*}\right) F_{1}^{\prime}\left(x_{0}\right)$ and is used in studying a method of collocation-type for nonlinear integral equations of Hammerstein type (see [6]). In general case, i.e., when both the operators $F_{i}, i=1,2$, are nonlinear, it means that $\mathcal{R}$, the range of the operator $I+F_{2}^{\prime}\left(x_{0}^{*}\right) F_{1}^{\prime}\left(x_{0}\right)$, is the whole space $X$. It is natural to ask if we can estimate the convergence rates for the sequences $\left\{x_{\alpha}^{h, \delta}\right\},\left\{x_{\alpha, n}^{h, \delta}\right\}$, when $\mathcal{R}$ is not the whole space $X$. For this purpose, only demanding that $\mathcal{R}$ contains a necessary element of $X$, the convergence rates of $\left\{x_{\alpha}^{h, \delta}\right\}$ and $\left\{x_{\alpha, n}^{h, \delta}\right\}$ are estimated in [4], [5] on the base of the zero value of the derivatives of higher order for $F_{1}$ and $F_{2}$ at $x_{0}$ and $x_{0}^{*}$, respectively. This result is formulated in the following theorem.

Theorem 1.1. (see [4] or [5]). Let the following conditions hold:

(i) $F_{1}$ is Fréchet differentiable at some neighbourhood $\mathcal{U}_{0}$ of $x_{0} s_{1}$-1-times if $s_{1}=\left[s_{1}\right]$, the integer part of $s_{1},\left[s_{1}\right]$-times if $s_{1} \neq\left[s_{1}\right]$, and $F_{2}$ is Fréchet differentiable at some neighbourhood $\mathcal{V}_{0}$ of $x_{0}^{*} s_{2}-1$-times, if $s_{2}=\left[s_{2}\right]$, [ $\left.s_{2}\right]$-times if $s_{2} \neq\left[s_{2}\right]$,

(ii) there exists a constant $L>0$ such that

$$
\begin{gathered}
\left\|F_{1}^{(k)}\left(x_{0}\right)-F_{1}^{(k)}(y)\right\| \leqslant L\left\|x_{0}-y\right\|, \quad \forall y \in \mathcal{U}_{0}, \\
\left\|F_{2}^{(k)}\left(x_{0}^{*}\right)-F_{2}^{(k)}\left(y^{*}\right)\right\| \leqslant L\left\|x_{0}^{*}-y^{*}\right\|, \quad \forall y^{*} \in \mathcal{V}_{0},
\end{gathered}
$$

for $F_{i}^{(k)}: k=s_{i}-1$ if $s_{i}=\left[s_{i}\right], k=\left[s_{i}\right]$ if $s_{i} \neq\left[s_{i}\right]$, and if $\left[s_{i}\right] \geqslant 3$, then $F_{1}^{(2)}\left(x_{0}\right)=\ldots=$ $F_{1}^{(k)}\left(x_{0}\right)=0$, and $F_{2}^{(2)}\left(x_{0}^{*}\right)=\ldots=F_{1}^{(k)}\left(x_{0}^{*}\right)=0$, 
(iii) there exists an element $x^{1} \in X$ such that

$$
\left(I+F_{2}^{\prime}\left(x_{0}^{*}\right)^{*} F_{1}^{\prime}\left(x_{0}\right)^{*}\right) x^{1}=F_{2}^{\prime}\left(x_{0}^{*}\right)^{*} U_{1}\left(x_{0}\right)-U_{2}\left(x_{0}^{*}\right),
$$

if $s_{1}=\left[s_{1}\right]$ then $L\left\|x^{1}\right\|<m_{1} s_{1}$ !, and if $s_{2}=\left[s_{2}\right]$ then $L\left\|F_{1}^{\prime}\left(x_{0}\right)^{*} x^{1}-U_{1}\left(x_{0}\right)\right\|<m_{2} s_{2}$ ! Then, if $\alpha$ is chosen such that $\alpha \sim(h+\varepsilon)^{\rho}, 0<\rho<1$, we have

$$
\begin{aligned}
\left\|x_{\omega}-x_{0}\right\| & =O\left((h+\varepsilon)^{\theta}\right), \\
\theta & =\min \left\{\theta_{1}, \frac{1-\rho+\theta_{2}}{s_{1}-1}\right\}, \\
\theta_{i} & =\min \left\{\frac{1-\rho}{s_{i}}, \frac{\rho}{s_{i}}\right\}, i=1,2 .
\end{aligned}
$$

In this paper, the convergence rates of $\left\{x_{\alpha}^{h, \delta}\right\}$ and $\left\{x_{\alpha, n}^{h, \delta}\right\}$ are established under much weaker conditions on $F_{i}, i=1,2$. These are the assumptions that $\mathcal{R}$ contains some element of $X$, and $F_{i}, i=1,2$, are inverse-strongly monotone, i.e.

$$
\begin{gathered}
\left\langle F_{1}(x)-F_{1}(y), x-y\right\rangle \geqslant \tilde{m}_{1}\left\|F_{1}(x)-F_{1}(y)\right\|^{2}, \quad x, y \in X, \\
\left\langle F_{2}\left(x^{*}\right)-F_{2}\left(y^{*}\right), x^{*}-y^{*}\right\rangle \geqslant \tilde{m}_{2}\left\|F_{2}\left(x^{*}\right)-F_{2}\left(y^{*}\right)\right\|^{2}, \quad x^{*}, y^{*} \in X^{*},
\end{gathered}
$$

where $\tilde{m}_{i}, i=1,2$, are some positive constants. Note that in [7] the inverse-strongly monotone property was used to estimate the convergence rates of the regularized solutions for ill-posed variational inequalities.

Below, by "a $\sim \mathrm{b}$ " we mean " $\mathrm{a}=\mathrm{O}(\mathrm{b})$ and $\mathrm{b}=\mathrm{O}(\mathrm{a})$ ".

\section{MAIN RESULTS}

Assume that the normalized dual mappings $U_{i}, i=1,2$, of the spaces $X$ and $X^{*}$ satisfy the following conditions (see [8])

$$
\begin{gathered}
\left\langle U_{i}\left(y_{1}^{i}\right)-U_{i}\left(y_{2}^{i}\right), y_{1}^{i}-y_{2}^{i}\right\rangle \geqslant m_{i}\left\|y_{1}^{i}-y_{2}^{i}\right\|^{s_{i}}, \quad m_{i}>0, s_{i} \geqslant 2, \\
\left\|U_{i}\left(y_{1}^{i}\right)-U_{i}\left(y_{2}^{i}\right)\right\| \leqslant c_{i}\left(R_{i}\right)\left\|y_{1}^{i}-y_{2}^{i}\right\|^{\nu_{i}}, \quad 0<\nu_{i} \leqslant 1,
\end{gathered}
$$

where $y_{1}^{i}, y_{2}^{i} \in X$ or $X^{*}$ on dependence of $i=1$ or 2 , respectively, and $c_{i}\left(R_{i}\right), R_{i}>0$, are the positive increasing functions on $R_{i}=\max \left\{\left\|y_{1}^{i}\right\|,\left\|y_{2}^{i}\right\|\right\}$.

The following theorem answers the question on convergence rates for $\left\{x_{\alpha}^{h, \delta}\right\}$.

Theorem 2.1. Assume that the following conditions hold:

(i) $F_{i}, i=1,2$, are inverse-strongly monotone and continuously Fréchet differentiable at some neighbourhoods $\mathcal{U}$ of $x_{0}$ and $\mathcal{V}$ of $x_{0}^{*}$, respectively, and

$$
\begin{gathered}
\left\|F_{1}(x)-F_{1}\left(x_{0}\right)-F_{1}^{\prime}\left(x_{0}\right)\left(x-x_{0}\right)\right\| \leqslant \tau_{1}\left\|F_{1}(x)-F_{1}\left(x_{0}\right)\right\|, \quad \forall x \in \mathcal{U}, \\
\left\|F_{2}\left(x^{*}\right)-F_{2}\left(x_{0}^{*}\right)-F_{2}^{\prime}\left(x_{0}^{*}\right)\left(x^{*}-x_{0}^{*}\right)\right\| \leqslant \tau_{2}\left\|F_{2}\left(x^{*}\right)-F_{2}\left(x_{0}^{*}\right)\right\|, \quad \forall x^{*} \in \mathcal{V},
\end{gathered}
$$

where $\tau_{i}, i=1,2$, are some positive constants, 
(ii) there exists an element $x^{1} \in X$ such that

$$
\left(I+F_{2}^{\prime}\left(x_{0}^{*}\right)^{*} F_{1}^{\prime}\left(x_{0}\right)^{*}\right) x^{1}=F_{2}^{\prime}\left(x_{0}^{*}\right)^{*} U_{1}\left(x_{0}\right)-U_{2}\left(x_{0}^{*}\right) .
$$

Then, if $\alpha$ is chosen such that $\alpha \sim(h+\delta)^{\rho}, 0<\rho<1$, we have

$$
\left\|x_{\alpha}^{h, \delta}-x_{0}\right\|=O\left((h+\delta)^{\theta / s_{1}}\right), \quad \theta=\min \{\rho / 2,1-\rho\} .
$$

Proof. Set

$$
A=m_{1}\left\|x_{\alpha}^{h, \delta}-x_{0}\right\|^{s_{1}}+m_{2}\left\|x_{\alpha}^{h, \delta, *}-x_{0}^{*}\right\|^{s_{2}}, \quad x_{\alpha}^{h, \delta, *}=F_{1, \alpha}^{h}\left(x_{\alpha}^{h, \delta}\right) .
$$

It is easy to see that $x_{0}$ is a solution of (1.1) iff $z_{0}=\left[x_{0}, x_{0}^{*}\right]$ is a solution of the system of following operator equations

$$
\begin{aligned}
F_{1}(x)-x^{*} & =0, \\
F_{2}\left(x^{*}\right)+x-f & =0 .
\end{aligned}
$$

Similarily, $x_{\alpha}^{h, \delta}$ is a regularized solution of the operator equation (1.2) iff $z_{\alpha}^{h, \delta}=\left[x_{\alpha}^{h, \delta}, x_{\alpha}^{h, \delta, *}\right]$ is a solution of the system of following equations

$$
\begin{array}{r}
F_{1}^{h}(x)+\alpha U_{1}(x)-x^{*}=0, \\
F_{2}^{h}\left(x^{*}\right)+\alpha U_{2}\left(x^{*}\right)+x-f_{\delta}=0 .
\end{array}
$$

Consider the space $Z=X \times X^{*}$ with the norm $\|z\|^{2}=\|x\|^{2}+\left\|x^{*}\right\|^{2}, z=\left[x, x^{*}\right], x \in X$, and $x^{*} \in X^{*}$. Then, the two above systems of equations can be written, respectively, in form of equations

$$
\begin{aligned}
\mathcal{A}(z) & =\bar{f} \\
\mathcal{A}_{\alpha}^{h}(z) & \equiv \mathcal{A}^{h}(z)+\alpha J(z)=\bar{f}_{\delta},
\end{aligned}
$$

where

$$
\begin{aligned}
\mathcal{A}(z) & =\left[F_{1}(x), F_{2}\left(x^{*}\right)\right]+\left[-x^{*}, x\right], \\
\mathcal{A}^{h}(z) & =\left[F_{1}^{h}(x), F_{2}^{h}\left(x^{*}\right)\right]+\left[-x^{*}, x\right], \\
J(z) & =\left[U_{1}(x), U_{2}\left(x^{*}\right)\right], \\
\bar{f} & =[0, f], \quad \bar{f}_{\delta}=\left[0, f_{\delta}\right] .
\end{aligned}
$$

It is easy to verify that $\mathcal{A}$ and $\mathcal{A}^{h}$ are the monotone operators from $Z$ to $Z^{*}=X^{*} \times X$, and the operator $J$ is the normalized duality mapping of the space $Z$. Hence, from (2.1), (2.3), (2.4) and the monotone property of $\mathcal{A}^{h}$ it implies that

$$
\begin{aligned}
A \leqslant & \left\langle J\left(z_{\alpha}^{h, \delta}\right)-J\left(z_{0}\right), z_{\alpha}^{h, \delta}-z_{0}\right\rangle \leqslant\left\langle J\left(z_{0}\right), z_{0}-z_{\alpha}^{h, \delta}\right\rangle \\
& +\frac{1}{\alpha}\left[\left\langle\bar{f}_{\delta}-\bar{f}, z_{\alpha}^{h, \delta}-z_{0}\right\rangle+\left\langle\mathcal{A}\left(z_{0}\right)-\mathcal{A}^{h}\left(z_{0}\right), z_{\alpha}^{h, \delta}-z_{0}\right\rangle\right] .
\end{aligned}
$$

It is not difficult to verify that

$$
\left\|\mathcal{A}^{h}(z)-\mathcal{A}(z)\right\| \leqslant \sqrt{2} h g(\|z\|) .
$$


Further, from (1.4) it follows

$$
\begin{aligned}
& \left\langle\mathcal{A}\left(z_{\alpha}^{h, \delta}\right)-\mathcal{A}\left(z_{0}\right), z_{\alpha}^{h, \delta}-z_{0}\right\rangle=\left\langle F_{1}\left(x_{\alpha}^{h, \delta}\right)-x_{\alpha}^{h, \delta, *}-\left(F_{1}\left(x_{0}\right)-x_{0}^{*}\right), x_{\alpha}^{h, \delta}-x_{0}\right\rangle \\
& +\left\langle F_{2}\left(x_{\alpha}^{h, \delta, *}\right)+x_{\alpha}^{h, \delta}-\left(F_{2}\left(x_{0}^{*}\right)+x_{0}\right), x_{\alpha}^{h, \delta, *}-x_{0}^{*}\right\rangle \\
& =\left\langle F_{1}\left(x_{\alpha}^{h, \delta}\right)-F_{1}\left(x_{0}\right), x_{\alpha}^{h, \delta}-x_{0}\right\rangle+\left\langle F_{2}\left(x_{\alpha}^{h, \delta, *}\right)-F_{2}\left(x_{0}^{*}\right), x_{\alpha}^{h, \delta, *}-x_{0}^{*}\right\rangle \\
& \geqslant \tilde{m}_{1}\left\|F_{1}\left(x_{\alpha}^{h, \delta}\right)-F_{1}\left(x_{0}\right)\right\|^{2}+\tilde{m}_{2}\left\|F_{2}\left(x_{\alpha}^{h, \delta, *}\right)-F_{2}\left(x_{0}^{*}\right)\right\|^{2} \\
& \geqslant \min \left\{\tilde{m}_{1}, \tilde{m}_{2}\right\} C^{2}, \quad C^{2}=\left\|F_{1}\left(x_{\alpha}^{h, \delta}\right)-F_{1}\left(x_{0}\right)\right\|^{2}+\left\|F_{2}\left(x_{\alpha}^{h, \delta, *}\right)-F_{2}\left(x_{0}^{*}\right)\right\|^{2} .
\end{aligned}
$$

On the other hand, from (2.3), (2.4)-(2.6) and the properties of $\mathcal{A}, \mathcal{A}^{h}, J, g$ we have

$$
\begin{aligned}
& \left\langle\mathcal{A}\left(z_{\alpha}^{h, \delta}\right)-\mathcal{A}\left(z_{0}\right), z_{\alpha}^{h, \delta}-z_{0}\right\rangle \leqslant\left\langle\bar{f}_{\delta}-\bar{f}, z_{\alpha}^{h, \delta}-z_{0}\right\rangle \\
& \quad+\alpha\left\langle J\left(z_{0}\right), z_{0}-z_{\alpha}^{h, \delta}\right\rangle+\left\langle\mathcal{A}\left(z_{\alpha}^{h, \delta}\right)-\mathcal{A}^{h}\left(z_{\alpha}^{h, \delta}\right), z_{\alpha}^{h, \delta}-z_{0}\right\rangle
\end{aligned}
$$

and $\left\{z_{\alpha}^{h, \delta}\right\}$ is bounded, as $(h+\delta) / \alpha \rightarrow 0$. Therefore,

$$
C^{2} \leqslant \frac{1}{\min \left\{\tilde{m}_{1}, \tilde{m}_{2}\right\}}\left[\delta+\alpha\left\|J\left(z_{0}\right)\right\|+\sqrt{2} h g\left(\left\|z_{\alpha}^{h, \delta}\right\|\right)\right]\left\|z_{\alpha}^{h, \delta}-z_{0}\right\| .
$$

Consequently, $C \leqslant O(\sqrt{h+\delta+\alpha})$. Hence,

$$
\begin{aligned}
\left\|F_{1}\left(x_{\alpha}^{h, \delta}\right)-F_{1}\left(x_{0}\right)\right\| & \leqslant O(\sqrt{h+\delta+\alpha}), \\
\left\|F_{2}\left(x_{\alpha}^{h, \delta, *}\right)-F_{2}\left(x_{0}^{*}\right)\right\| & \leqslant O(\sqrt{h+\delta+\alpha}) .
\end{aligned}
$$

Now, we shall estimate the value $\left\langle J\left(z_{0}\right), z_{0}-z_{\alpha}^{h, \delta}\right\rangle$. For this purpose, set $x^{2}=U_{1}\left(x_{0}\right)-$ $F_{1}^{\prime}\left(x_{0}\right)^{*} x^{1}$. From condition (ii) of the theorem it follows that $x^{1}$ and $x^{2}\left(\in X^{*}\right)$ satisfy the system of following equalities

$$
\begin{aligned}
& F_{1}^{\prime}\left(x_{0}\right)^{*} x^{1}+x^{2}=U_{1}\left(x_{0}\right) \\
& F_{2}^{\prime}\left(x_{0}^{*}\right)^{*} x^{2}-x^{1}=U_{2}\left(x_{0}^{*}\right)
\end{aligned}
$$

By virtue of

$$
\begin{aligned}
\left\langle J\left(z_{0}\right), z_{0}-z_{\alpha}^{h, \delta}\right\rangle= & \left\langle U_{1}\left(x_{0}\right), x_{0}-x_{\alpha}^{h, \delta}\right\rangle+\left\langle U_{2}\left(x_{0}^{*}\right), x_{0}^{*}-x_{\alpha}^{h, \delta, *}\right\rangle \\
= & \left\langle F_{1}^{\prime}\left(x_{0}\right)^{*} x^{1}+x^{2}, x_{0}-x_{\alpha}^{h, \delta}\right\rangle+\left\langle F_{2}^{\prime}\left(x_{0}^{*}\right)^{*} x^{2}-x^{1}, x_{0}^{*}-x_{\alpha}^{h, \delta, *}\right\rangle \\
= & \left\langle x_{\alpha}^{h, \delta, *}-x_{0}^{*}-F_{1}^{\prime}\left(x_{0}\right)\left(x_{\alpha}^{h, \delta}-x_{0}\right), x^{1}\right\rangle \\
& +\left\langle x_{0}-x_{\alpha}^{h, \delta}-F_{2}^{\prime}\left(x_{0}^{*}\right)\left(x_{\alpha}^{h, \delta, *}-x_{0}^{*}\right), x^{2}\right\rangle \\
= & \left\langle F_{1}\left(x_{\alpha}^{h, \delta}\right)-F_{1}\left(x_{0}\right)-F_{1}^{\prime}\left(x_{0}\right)\left(x_{\alpha}^{h, \delta}-x_{0}\right), x^{1}\right\rangle \\
& +\alpha\left\langle U_{1}\left(x_{\alpha}^{h, \delta}\right), x^{1}\right\rangle+\left\langle F_{1}^{h}\left(x_{\alpha}^{h, \delta}\right)-F_{1}\left(x_{\alpha}^{h, \delta}\right), x^{1}\right\rangle \\
& +\left\langle F_{2}\left(x_{\alpha}^{h, \delta, *}\right)-F_{2}\left(x_{0}^{*}\right)-F_{2}^{\prime}\left(x_{0}^{*}\right)\left(x_{\alpha}^{h, \delta, *}-x_{0}^{*}\right), x^{2}\right\rangle \\
& \left.+\left\langle\alpha U_{2}\left(x_{\alpha}^{h, \delta, *}\right)+f-f_{\delta}, x^{2}\right\rangle+\left\langle F_{2}^{h}\left(x_{\alpha}^{h, \delta, *}\right)-F_{(} x_{\alpha}^{h, \delta, *}\right), x^{2}\right\rangle,
\end{aligned}
$$

we have

$$
\begin{aligned}
& \left\langle J\left(z_{0}\right), z_{0}-z_{\alpha}^{h, \delta}\right\rangle \leqslant \max \left\{\tau_{1}\left\|x^{1}\right\|, \tau_{2}\left\|x^{2}\right\|\right\} \times \\
& \quad\left(\left\|F_{1}\left(x_{\alpha}^{h, \delta}\right)-F_{1}\left(x_{0}\right)\right\|+\left\|F_{2}\left(x_{\alpha}^{h, \delta, *}\right)-F_{2}\left(x_{0}^{*}\right)\right\|\right)+O(h+\delta+\alpha) .
\end{aligned}
$$


Thus, for sufficiently small $h, \delta, \alpha(h+\delta+\alpha<1)$ from (2.5)-(2.7) we have got

$$
A \leqslant O\left((h+\delta)^{1-\rho}\right)+O(\sqrt{h+\delta+\alpha}),
$$

It means that

$$
\left\|x_{\alpha}^{h, \delta}-x_{0}\right\|=O\left((h+\delta)^{\theta / s_{1}}\right)
$$

Theorem is proved.

Theorem 2.2. Assume that the conditions of Theorem 2.1 hold, and $\alpha$ is chosen such that $\alpha \sim\left(h+\delta+\gamma_{n}\right)^{\rho}, 0<\rho<1$, where

$$
\gamma_{n}=\max \left\{\left\|\left(I-P_{n}\right) x_{0}\right\|,\left\|\left(I-P_{n}\right) f\right\|,\left\|\left(I-P_{n}\right) x^{1}\right\|,\left\|\left(I^{*}-P_{n}^{*}\right) x_{0}^{*}\right\|,\left\|\left(I^{*}-P_{n}^{*}\right) x^{2}\right\|\right\},
$$

and $I^{*}$ denotes the identity operator in $X^{*}$. Then,

$$
\begin{aligned}
\left\|x_{\alpha, n}^{h, \delta}-x_{0}\right\| & =O\left((h+\delta)^{\eta}+\gamma_{n}^{\mu}\right), \\
\eta & =\min \left\{\frac{1-\rho}{s_{1}}, \frac{\rho}{2 s_{1}}\right\}, \\
\mu & =\min \left\{\eta, \frac{\nu_{1}}{s_{1}}, \frac{\nu_{2}}{s_{1}}\right\} .
\end{aligned}
$$

Proof. Set

$$
B=m_{1}\left\|x_{\alpha, n}^{h, \delta}-x_{0, n}\right\|^{s_{1}}+m_{2}\left\|x_{\alpha, n}^{h, \delta, *}-x_{0, n}^{*}\right\|^{s_{2}},
$$

with $x_{0, n}=P_{n} x_{0}, x_{\alpha, n}^{h, \delta, *}=F_{1, \alpha, n}^{h}\left(x_{\alpha, n}^{h, \delta}\right)$, and $x_{0, n}^{*}=P_{n}^{*} x_{0}^{*}$. It is easy to see that $x_{\alpha, n}^{h, \delta}$ is a solution of (1.3) iff $x_{\alpha, n}^{h, \delta}$ and $x_{\alpha, n}^{h, \delta, *}$ are the solutions of the system of following equations

$$
\begin{aligned}
F_{1, n}^{h}(x)+\alpha U_{1}^{n}(x)-x^{*} & =0, \\
F_{2 n}^{h}\left(x^{*}\right)+\alpha U_{2}^{n}\left(x^{*}\right)+x-f_{\delta, n} & =0,
\end{aligned}
$$

with $U_{1}^{n}=P_{n}^{*} U_{1} P_{n}, U_{2}^{n}=P_{n} U_{2} P_{n}^{*}, F_{1, n}^{h}=P_{n}^{*} F_{1}^{h} P_{n}, F_{2, n}^{h}=P_{n} F_{2}^{h} P_{n}^{*}$, and $f_{\delta, n}=P_{n} f_{\delta}$. As in the proof of theorem 2.1, $z_{\alpha, n}^{h, \delta}:=\left[x_{\alpha, n}^{h, \delta}, x_{\alpha, n}^{h, \delta, *}\right]$ is the solution of the following operator equation

$$
\mathcal{A}_{\alpha, n}^{h}(z) \equiv \mathcal{A}_{n}^{h}(z)+\alpha J^{n}(z)=\bar{f}_{\delta, n},
$$

where

$$
\begin{aligned}
\mathcal{A}_{n}^{h}(z) & =\left[F_{1, n}^{h}(x), F_{2, n}^{h}\left(x^{*}\right)\right]+\left[-x^{*}, x\right], \\
J^{n}(z) & =\left[U_{1}^{n}(x), U_{2}^{n}\left(x^{*}\right)\right], \quad \bar{f}_{\delta, n}=\left[0, f_{\delta, n}\right] .
\end{aligned}
$$

The operators $\mathcal{A}_{n}^{h}$ and $\mathcal{A}_{n}$, defined by $\mathcal{A}_{n}(z)=\left[F_{1, n}(x), F_{2, n}\left(x^{*}\right)\right]+\left[-x^{*}, x\right], F_{1, n}=P_{n}^{*} F_{1} P_{n}, F_{2, n}=$ $P_{n} F_{2} P_{n}^{*}$, are the monotone operators, and act from $Z_{n}:=X_{n} \times X_{n}^{*}$ into $Z_{n}^{*}$, and $J^{n}$ is the normalized duality mapping of the space $Z_{n}$.

From (2.8) we obtain

$$
\begin{aligned}
\mathcal{A}_{n}\left(z_{\alpha, n}^{h, \delta}\right)-\mathcal{A}_{n}\left(z_{0, n}\right) & +\alpha\left[J^{n}\left(z_{\alpha, n}^{h, \delta}\right)-J^{n}\left(z_{0, n}\right)\right]=\bar{f}_{\delta, n}+ \\
& \mathcal{A}_{n}\left(z_{\alpha, n}^{h, \delta}\right)-\mathcal{A}_{n}^{h}\left(z_{\alpha, n}^{h, \delta}\right)-\mathcal{A}_{n}\left(z_{0, n}\right)-\alpha J^{n}\left(z_{0, n}\right) .
\end{aligned}
$$


Therefore, from (1.4) and the properties of the projections $P_{n}, P_{n}^{*}$ it implies that

$$
\begin{aligned}
& \left\langle\mathcal{A}_{n}\left(z_{\alpha, n}^{h, \delta}\right)-\mathcal{A}_{n}\left(z_{0, n}\right), z_{\alpha, n}^{h, \delta}-z_{0, n}\right\rangle=\left\langle F_{1}\left(x_{\alpha, n}^{h, \delta}\right)-F_{1}\left(x_{0, n}\right), x_{\alpha, n}^{h, \delta}-x_{0, n}\right\rangle \\
& +\left\langle F_{2}\left(x_{\alpha, n}^{h, \delta, *}\right)-F_{2}\left(x_{0, n}^{*}\right), x_{\alpha, n}^{h, \delta, *}-x_{0, n}^{*}\right\rangle \\
& \geqslant \tilde{m}_{1}\left\|F_{1}\left(x_{\alpha, n}^{h, \delta}\right)-F_{1}\left(x_{0, n}\right)\right\|^{2}+\tilde{m}_{2}\left\|F_{2}\left(x_{\alpha, n}^{h, \delta, *}\right)-F_{2}\left(x_{0, n}^{*}\right)\right\|^{2} \\
& \geqslant \min \left\{\tilde{m}_{1}, \tilde{m}_{2}\right\} C_{n}^{2}, \quad C_{n}^{2}=\left\|F_{1}\left(x_{\alpha, n}^{h, \delta}\right)-F_{1}\left(x_{0, n}\right)\right\|^{2}+\left\|F_{2}\left(x_{\alpha, n}^{h, \delta, *}\right)-F_{2}\left(x_{0, n}^{*}\right)\right\|^{2} .
\end{aligned}
$$

On the other hand, from (2.8), (2.9) we also obtain

$$
\begin{aligned}
\mathcal{A}_{n}^{h}\left(z_{\alpha, n}^{h, \delta}\right)-\mathcal{A}_{n}^{h}\left(z_{0, n}\right) & +\alpha\left[J^{n}\left(z_{\alpha, n}^{h, \delta}\right)-J^{n}\left(z_{0, n}\right)\right]=\bar{f}_{\delta, n} \\
& -\mathcal{A}_{n}^{h}\left(z_{0, n}\right)-\alpha J^{n}\left(z_{0, n}\right) .
\end{aligned}
$$

Hence, on the base of the property of $J$ and (2.11) we can write

$$
\begin{aligned}
B & \leqslant \frac{1}{\alpha}\left\langle\bar{f}_{\delta}-\bar{f}-\alpha J\left(z_{0, n}\right), z_{\alpha, n}^{h, \delta}-z_{0, n}\right\rangle \\
& +\frac{1}{\alpha}\left\langle\mathcal{A}\left(z_{0}\right)-\mathcal{A}^{h}\left(z_{0, n}\right), z_{\alpha, n}^{h, \delta}-z_{0, n}\right\rangle \\
& \leqslant \frac{1}{\alpha}\left[\delta+\left\|\mathcal{A}\left(z_{0}\right)-\mathcal{A}\left(z_{0, n}\right)\right\|+h g\left(\left\|z_{0, n}\right\|\right)\right]\left\|z_{\alpha, n}^{h, \delta}-z_{0, n}\right\| \\
& +\left\langle J^{n}\left(z_{0, n}\right), z_{0, n}-z_{\alpha, n}^{h, \delta}\right\rangle .
\end{aligned}
$$

Moreover, using the continously Fréchet differentiable property of $F_{1}, F_{2}$ and the definition of $\gamma_{n}$ we can also write

$$
\begin{aligned}
\left\|\mathcal{A}\left(z_{0, n}\right)-\mathcal{A}\left(z_{0}\right)\right\| & \leqslant\left(\left\|F_{1}\left(x_{0, n}\right)-F_{1}\left(x_{0}\right)\right\|^{2}\right. \\
& \left.+\left\|F_{2}\left(x_{0, n}^{*}\right)-F_{2}\left(x_{0}^{*}\right)\right\|^{2}\right)^{1 / 2}+\sqrt{2} \gamma_{n} \\
& \leqslant\left(\max \left\{\tilde{c}_{1}, \tilde{c}_{2}\right\}+\sqrt{2}\right) \gamma_{n}
\end{aligned}
$$

where $\tilde{c}_{1}=\max _{0 \leqslant t \leqslant 1}\left\|F_{1}^{\prime}\left(x_{0}+t\left(x_{0, n}-x_{0}\right)\right)\right\|$ and $\tilde{c}_{2}=\max _{0 \leqslant t \leqslant 1}\left\|F_{2}^{\prime}\left(x_{0}^{*}+t\left(x_{0, n}^{*}-x_{0}^{*}\right)\right)\right\|$. Consequently, $\left\{z_{\alpha, n}^{h, \delta}\right\}$ is bounded, when $\left(h+\delta+\gamma_{n}\right) / \alpha \rightarrow 0$. By virtue of (2.10) we have

$$
\begin{aligned}
& \left\langle\mathcal{A}_{n}\left(z_{\alpha, n}^{h, \delta}\right)-\mathcal{A}_{n}\left(z_{0, n}\right), z_{\alpha, n}^{h, \delta}-z_{0, n}\right\rangle \leqslant\left\langle\bar{f}_{\delta, n}-\mathcal{A}_{n}\left(z_{0, n}\right), z_{\alpha, n}^{h, \delta}-z_{0, n}\right\rangle \\
& \quad+\left\langle\mathcal{A}_{n}\left(z_{\alpha, n}^{h, \delta}\right)-\mathcal{A}_{n}^{h}\left(z_{\alpha, n}^{h, \delta}\right)-\alpha J^{n}\left(z_{0, n}\right), z_{\alpha, n}^{h, \delta}-z_{0, n}\right\rangle \\
& \quad \leqslant\left\langle\bar{f}_{\delta}-\bar{f}+\mathcal{A}\left(z_{0}\right)-\mathcal{A}\left(z_{0, n}\right), z_{\alpha, n}^{h, \delta}-z_{0, n}\right\rangle \\
& +\left\langle\mathcal{A}_{n}\left(z_{\alpha, n}^{h, \delta}\right)-\mathcal{A}_{n}^{h}\left(z_{\alpha, n}^{h, \delta}\right)-\alpha J\left(z_{0, n}\right), z_{\alpha, n}^{h, \delta}-z_{0, n}\right\rangle \\
& \left.\leqslant O\left(h+\delta+\alpha+\gamma_{n}\right) \| z_{0, n}-z_{\alpha, n}^{h, \delta}\right\rangle \| .
\end{aligned}
$$

Therefore, $\tilde{C}_{n} \leqslant O\left(\sqrt{h+\delta+\alpha+\gamma_{n}}\right)$. Hence,

$$
\begin{aligned}
\left\|F_{1}\left(x_{\alpha, n}^{h, \delta}\right)-F_{1}\left(x_{0, n}\right)\right\| & \leqslant O\left(\sqrt{h+\delta+\alpha+\gamma_{n}}\right), \\
\left\|F_{2}\left(x_{\alpha, n}^{h, \delta, *}\right)-F_{2}\left(x_{0, n}^{*}\right)\right\| & \leqslant O\left(\sqrt{h+\delta+\alpha+\gamma_{n}}\right) .
\end{aligned}
$$


Now, we obtain the esimation for $\left\langle J^{n}\left(z_{0, n}\right), z_{0, n}-z_{\alpha, n}^{h, \delta}\right\rangle$. From $(2.2),(2.8)$ and the condition of the theorem we have got

$$
\begin{aligned}
& \left\langle J^{n}\left(z_{0, n}\right), z_{0, n}-z_{\alpha, n}^{h, \delta}\right\rangle=\left\langle J\left(z_{0, n}\right), z_{0, n}-z_{\alpha, n}^{h, \delta}\right\rangle \\
& =\left\langle J\left(z_{0, n}\right)-J\left(z_{0}\right), z_{0, n}-z_{\alpha, n}^{h, \delta}\right\rangle+\left\langle J\left(z_{0}\right), z_{0, n}-z_{\alpha, n}^{h, \delta}\right\rangle \\
& \leqslant \bar{C} \gamma_{n}^{\nu}\left\|z_{\alpha, n}^{h, \delta}-z_{0, n}\right\|+\left\langle F_{1}^{\prime}\left(x_{0}\right)^{*} x^{1}+x^{2}, x_{0, n}-x_{\alpha, n}^{h, \delta}\right\rangle \\
& \quad+\left\langle F_{2}^{\prime}\left(x_{0}^{*}\right)^{*} x^{2}-x^{1}, x_{0, n}^{*}-x_{\alpha, n}^{h, \delta, *}\right\rangle \\
& \leqslant \bar{C} \\
& \gamma_{n}^{\nu}\left\|z_{\alpha, n}^{h, \delta}-z_{0, n}\right\|+\left\langle x^{1}, x_{\alpha, n}^{h, \delta, *}-x_{0, n}^{*}-F_{1}^{\prime}\left(x_{0}\right)\left(x_{\alpha, n}^{h, \delta}-x_{0, n}\right)\right\rangle \\
& +\left\langle x^{2}, x_{0, n}-x_{\alpha, n}^{h, \delta}-F_{2}^{\prime}\left(x_{0}^{*}\right)\left(x_{\alpha, n}^{h, \delta, *}-x_{0, n}^{*}\right)\right\rangle
\end{aligned}
$$

where $\bar{C}$ is some positive constant, and $\nu=\min \left\{\nu_{1}, \nu_{2}\right\}$. Obviously,

$$
\begin{aligned}
& \left\langle x^{1}, x_{\alpha, n}^{h, \delta, *}-x_{0, n}^{*}-F_{1}^{\prime}\left(x_{0}\right)\left(x_{\alpha, n}^{h, \delta}-x_{0, n}\right)\right\rangle=\left\langle x^{1}, F_{1, n}^{h}\left(x_{\alpha, n}^{h, \delta}\right)+\alpha U_{1}^{n}\left(x_{\alpha, n}^{h, \delta}\right)-x_{0, n}^{*}\right\rangle \\
& +\left\langle x^{1},-F_{1}^{\prime}\left(x_{0}\right)\left(x_{\alpha, n}^{h, \delta}-x_{0}\right)+F_{1}^{\prime}\left(x_{0}\right)\left(x_{0, n}-x_{0}\right)\right\rangle \\
& =\left\langle x_{n}^{1}, F_{1}\left(x_{\alpha, n}^{h, \delta}\right)-F_{1}\left(x_{0}\right)-F_{1}^{\prime}\left(x_{0}\right)\left(x_{\alpha, n}^{h, \delta}-x_{0}\right)\right\rangle \\
& \quad+\alpha\left\langle x^{1}, U_{1}^{n}\left(x_{\alpha, n}^{h, \delta}\right)\right\rangle+\left\langle x^{1}, F_{1}^{\prime}\left(x_{0}\right)\left(x_{0, n}-x_{0}\right)\right\rangle \\
& \quad+\left\langle\left(I-P_{n}\right) x^{1},-F_{1}^{\prime}\left(x_{0}\right)\left(x_{\alpha, n}^{h, \delta}-x_{0}\right)\right\rangle+\left\langle x_{n}^{1}, F_{1}^{h}\left(x_{\alpha, n}^{h, \delta}\right)-F_{1}\left(x_{\alpha, n}^{h, \delta}\right)\right\rangle \\
& \leqslant \tau_{1}\left\|x_{n}^{1}\right\|\left\|F_{1}\left(x_{\alpha, n}^{h, \delta}\right)-F_{1}\left(x_{0}\right)\right\|+O\left(h+\alpha+\gamma_{n}\right),
\end{aligned}
$$

where $x_{n}^{1}=P_{n} x^{1}$. By the argument, we also obtain the estimate

$$
\begin{gathered}
\left\langle x^{2}, x_{0, n}-x_{\alpha, n}^{h, \delta}-F_{2}^{\prime}\left(x_{0}^{*}\right)\left(x_{\alpha, n}^{h, \delta, *}-x_{0, n}^{*}\right)\right\rangle \leqslant \tau_{2}\left\|x_{n}^{2}\right\|\left\|F_{2}\left(x_{\alpha, n}^{h, \delta, *}\right)-F_{2}\left(x_{0}^{*}\right)\right\| \\
+O\left(h+\delta+\alpha+\gamma_{n}\right) .
\end{gathered}
$$

Therefore,

$$
\left\langle J^{n}\left(z_{0}\right), z_{0}-z_{\alpha}^{h, \delta}\right\rangle \leqslant O\left(\gamma_{n}^{\nu}\right)+O\left(\sqrt{h+\delta+\gamma_{n}+\alpha}\right) .
$$

Thus, from (2.12) and the properties of $\mathcal{A}^{h}, J$ it follows

$$
B \leqslant O\left(\left(h+\delta+\gamma_{n}\right)^{1-\rho}+\gamma_{n}^{\nu}+O\left(\left(h+\delta+\gamma_{n}\right)^{\rho / 2}\right) .\right.
$$

Consequently,

$$
\left\|x_{\alpha, n}^{h, \delta}-x_{0}\right\|=O\left((h+\delta)^{\eta}+\gamma_{n}^{\mu}\right)
$$

Theorem is proved.

Example 1. Consider the simple example, when $X \equiv X^{*}=\mathbf{E}^{2}$, the Euclid space, and

$$
F_{1}=\left[\begin{array}{cc}
1 & -1 \\
1 & 0
\end{array}\right], F_{2}=\left[\begin{array}{cc}
0 & -1 \\
1 & 1
\end{array}\right], x=\left(x_{1}, x_{2}\right) .
$$

It is easy to verify that $\left\langle F_{1} x, x\right\rangle=x_{1}^{2} \geqslant 0$, and $\left\langle F_{2} x, x\right\rangle=x_{2}^{2} \geqslant 0 \forall x \in \mathbf{E}^{2}$. It means that $F_{i}, i=1,2$, are monotone. Equation (1.1) has the form $0 x_{1}=f_{1}, \quad 2 x_{1}=f_{2}$ with $f=\left(f_{1}, f_{2}\right)$. Obviously, this system of equations has a unique solution when $f=\left(0, f_{2}\right)$ for arbitrary $f_{2}$. When $f_{\delta}=\left(f_{1}^{\delta}, f_{2}\right)$ with $f_{1}^{\delta} \neq 0$ equation (1.1) in this case there isn't a solution. So, equation 
(1.1) with the monotone operators $F_{1}, i=1,2$, in general is ill-posed. On the other hand, equation $\mathcal{A}(z)=\bar{f}$ for $z=\left(x_{1}, x_{2}, x_{1}^{*}, x_{2}^{*}\right)$ is the system of 4 linear equations with the matrix

$$
\mathcal{A}=\left[\begin{array}{cccc}
1 & -1 & -1 & 0 \\
1 & 0 & 0 & -1 \\
1 & 0 & 0 & -1 \\
0 & 1 & 1 & 1
\end{array}\right]
$$

having $\operatorname{det} \mathcal{A}=0$. Consequently, the system of equations is also ill-posed.

\section{REFERENCES}

[1] N. Buong, On solutions of the equations of Hammerstein type in Banach spaces, $Z h$. Vychisl. Matematiki i Matem. Fiziki 25 (8) (1985) 1256-1280 (in Russian).

[2] N. Buong, On solution of Hammerstein's equation with monotone perturbations, Vietnamese Math. Journal 3 (1985) 28-32.

[3] N. Buong, On approximate solution for operator equations of Hammerstein type, J. of Comput. and Applied Math. 75 (1996) 77-86.

[4] N. Buong, Convergence rates in regularization for Hammerstein equations, Zh. Vychisl. Matematiki i Matem. Fiziki 39 (4) (1999) 3-7.

[5] N. Buong, Convergence rates in regularization for the case of monotone perturbations, Ukrainian Math. Zh. 52 (2) (2000) 285-293.

[6] S. Kumar, Superconvergence of a Collocation - type Method for Hammerstein Equations, IMA Journal of Numerical Analysis 7 (1987) 313-325.

[7] F. Liu and M. Z. Nashed, Regularization of nonlinear ill-posed variational inequalities and convergence rates, Set-Valued Analysis 6 (1998) 313-344.

[8] I. P. Ryazantseva, On an algorithm for solving nonlinear monotone equations with unknow estimate input errors, Zh. Vychisl. Matematiki i Matem. Fiziki 29 (1989) 1572-1576 (in Russian).

[9] M. M. Vainberg, Variational method and method of monotone operators, Nauka, Moscow 1972 (in Russian).

Received on April 6 - 2006

Revised on November 14 - 2006 